\title{
Manganese and cobalt levels in hair and urine of RUDN University first-year students
}

\author{
Anatoly A. Kirichuk, ${ }^{*}$ \\ Peoples' Friendship University of Russia (RUDN University), Faculty of Ecology, 6 Miklukho- \\ Maklaya St, Moscow, 117198, Russian Federation
}

\begin{abstract}
The objective of the present study was to assess hair and urinary levels of cobalt $(\mathrm{Co})$ and manganese $(\mathrm{Mn})$ in foreign students of RUDN University in comparison to the Russian counterparts. A total of 272 first-year students of the RUDN University originating from Russia, Asia, Middle East, Africa, and Latin America were enrolled in the study. Evaluation of $\mathrm{Mn}$ and Co levels in urine and hair was performed using inductively-coupled plasma mass-spectrometry. The obtained data demonstrate that hair cobalt levels were found to be the highest in students originating from Russia and Africa. Similarly, urinary Co levels in subjects from Asia, Middle East, and Latin America were significantly lower than those in the Russian students. The patterns of manganese status were generally similar to those observed for cobalt. Particularly, the lowest hair Mn content was detected in subjects originating from Middle East. Manganese level in urine of students from Africa was significantly higher than that in subjects from Asia, Middle East, and Latin America, but not Russia. It is proposed that specific national dietary patterns as well as geochemistry of Mn and Co may underlie the observed differences.
\end{abstract}

\section{Introduction}

Essential trace elements participate in a plethora of metabolic processes thus being required for organism functioning [1]. Micronutrient deficiency is considered a significant public health concern due to both high medio-social outcome and wide prevalence exceeding 2 billion of people worldwide [2]. However, data on micronutrient deficiency are insufficient and highly contradictory for different regions. Systematic data exist on only certain micronutrients including vitamins, as well as iron, zinc, iodine, selenium [2], whereas the prevalence of inadequate intake of other essential elements is underestimated.

Manganese [3] and cobalt [4] are also considered as essential metals, the particular clinical features of their deficiency are not estimated. At the same time, both Mn [3] and Co [5] overload may exert toxic effects. Particularly, the health effects of manganese are explained by U-shaped relationship, when both deficiency and toxicity are associated with adverse health effects [6]. At the same time, data on geographic variability of cobalt and manganese status are absent.

\footnotetext{
*Corresponding author: Kirichuk-aa@ rudn.ru
} 
RUDN University is characterized by the highest number of foreign students from various regions including Middle East, Asia, Africa, and Latin America. Therefore, the objective of the present study was to assess hair and urinary levels of cobalt and manganese in foreign students of RUDN University in comparison to the Russian counterparts.

\section{Materials and methods}

A total of 272 first-year students (40\% males, $60 \%$ females) of the RUDN University originating from Russia $(n=65)$, Asia $(n=57)$, Middle East $(n=84)$, Africa $(n=40)$, and Latin America $(n=28)$. Anthropometric examination as well as sample collection was performed during the first medical examination. The overall mean age of the examinees was $22.4 \pm 4.3$ years. Due to group variability all comparisons were adjusted for age and gender values. The protocol of the current study was approved by the Institutional Ethics Committee (RUDN University, Moscow, Russia). All procedures were performed in agreement with the principles set in the Declaration of Helsinki and its later amendments (2013).

Evaluation of manganese $(\mathrm{Mn})$ and cobalt $(\mathrm{Co})$ levels in urine and hair after microwave digestion was performed using inductively-coupled plasma mass-spectrometry (ICP-MS) at NexION 300D (PerkinElmer Inc., USA). Laboratory quality control was performed using certified reference materials of human hair and urine.

The obtained data were processed using Statistica 10.0 (Statsoft, OK, USA) software. Due to skewed distribution as assessed by Shapiro-Wilk test, median and interquartile range (IQR) boundaries were used as descriptive statistics for hair and urinary metal levels. In order to account for variability in age and gender, assessment of the group difference was performed using analysis of covariance (ANCOVA) with Fisher's LSD post-hoc test at the level of significance of $p<0.05$. Multiple regression analysis with adjustment for age and gender was performed in order to evaluate the association between hair Mn and Co levels (dependent variables) and urinary metal levels, as well as the region of origin (independent variables).

\section{Results}

The obtained data demonstrate that hair cobalt levels were found to be the highest in students originating from Russia and Africa. Particularly, hair Co content in students from Asia, Middle East, and Latin America were 53\%, 79\%, and 42\% lower as compared to the respective values in Russian examinees. In turn, the African student values exceeded the respective ones in examinees from Asia by a factor of more than four. No significant group difference in Co levels in hair between Russian and African students was observed.

Generally, the difference in urinary cobalt levels followed similar patterns. Particularly, urinary Co levels in subjects from Asia, Middle East, and Latin America were 40\%, 34\%, and $26 \%$ lower than those in the Russian students. Urinary Co concentration in African students exceeded the respective values in subjects originating from Asia and Latin America by $60 \%$ and $31 \%$. It is notable that the values of urinary Co levels were nearly similar in African and Russian students.

It is notable that the patterns of manganese status were generally similar to those observed for cobalt. Specifically, the lowest hair Mn content was detected in subjects originating from Middle East, being lower than that in students from Russia, Asia, Africa, and Latin America by 76\%, 44\%, 78\%, and 69\%, respectively. Hair Mn content in subjects originating from Africa and Latin America also exceeded the respective values found in the 
group of Asian students by $161 \%$ and $84 \%$. No significant group difference was observed in hair Mn content in students from Russia, Africa, and Latin America was observed.

Manganese level in urine of students from Africa was significantly higher than that in subjects from Asia, Middle East, and Latin America by 43\%, 29\%, and 25\%, but did not differ significantly from the Russian values. At the same time, the values of urinary Co concentrations in Russian students exceeded those in subjects from Asia by $26 \%$.

Table 1. Hair $(\mu \mathrm{g} / \mathrm{g})$ and urinary $(\mathrm{ng} / \mathrm{ml})$ Co and Mn levels of Russian and foreign students of RUDN University

\begin{tabular}{|l|l|l|l|l|}
\hline Region & Hair Co & Urine Co & Hair Mn & Urine Mn \\
\hline \multirow{3}{*}{ Russia } & 0.019 & 1.16 & 0.843 & 0.891 \\
\hline & $(0.009-0.049)$ & $(0.728-2.057)$ & $(0.335-1.179)$ & $(0.603-1.309)$ \\
Asia & $0.009^{1}$ & $0.705^{1}$ & 0.353 & $0.706^{1}$ \\
\hline & $(0.004-0.017)$ & $(0.53-1.017)$ & $(0.238-1.037)$ & $(0.542-1.011)$ \\
\hline \multirow{2}{*}{ East } & $0.004^{1}$ & $0.768^{1}$ & $0.200^{1,2}$ & 0.784 \\
Africa & $(0.003-0.007)$ & $(0.54-0.973)$ & $(0.155-0.354)$ & $(0.564-1.162)$ \\
\hline & $0.018^{3}$ & $1.127^{2}$ & $0.923^{2,3}$ & $1.01^{2,3}$ \\
Latin America & $(0.012-0.031)$ & $(0.767-1.717)$ & $(0.604-1.411)$ & $(0.723-1.343)$ \\
\hline Data are expressed as median (IQR); $1,2,3,4-$ significant group difference as compared to the \\
respective values in students from Russia (1), Asia (2), Middle East (3), and Africa (4) at p < 0.05, \\
respectively (ANCOVA Fisher's LSD post-hoc) \\
\hline
\end{tabular}

Multiple regression analysis demonstrated that hair Co level was inversely associated with male gender $(\beta=-0.374 ; p<0.001)$ and urinary metal excretion $(\beta=-0.131 ; p 0.032)$. At the same time, no significant relationship between the country of origin and hair Co content was revealed. The overall model incorporating age, gender, geographic region, as well as urinary Co concentrations accounted for $15 \%$ of hair Co variability $(p<0.001)$.

In contrast, no significant relationship between hair and urinary Mn levels was observed $(\beta=0.032 ; p=0.545)$. As in the case of cobalt, male gender was found to be a negative predictor of hair Mn content $(\beta=-0.435 ; \mathrm{p}<0.001 *)$. It is also notable that African origin was significantly associated with higher hair Mn levels $(\beta=0.207 ; p=0.046)$. Generally, the overall model was significantly associated with up to $24 \%$ of hair Mn variability ( $\mathrm{p}<$ $0.001)$.

\section{Conclusion}

The obtained data demonstrate that the lowest levels of cobalt and manganese in RUDN University students were found in subjects originating from Asia and especially Middle East. In turn, the highest levels were observed in the Russian and African examinees. At the same time, the obtained values of hair Co content in Russian students also indicate suboptimal Co status, being lower than the reference ranges for Co estimated as 0.07-0.50 $\mu \mathrm{g} / \mathrm{g}$ [7]. The obtained data on hair Mn levels in Russian students corresponds to the reference limit of 0.29-1.76 $\mu \mathrm{g} / \mathrm{g}$ [7]. Lower levels of hair Co and $\mathrm{Mn}$ in newcomers from Middle East are in agreement with the earlier data [8].

Reduced hair Co content may be hypothetically indicative of suboptimal intake of vitamin B12, being a biologically active form of cobalt [9]. In this view, low hair Co levels correspond to high prevalence of vitamin B12 deficiency in subjects living in Middle East and Asia [10]. Reduced dietary intake of cobalt [11] and vitamin B12 [12] with low-meat diets may underlie the observed deficiencies. 
At the same time, the particular causes of the observed low Mn status in students from Middle East are unclear. Cereals and leafy vegetables are considered as prior sources of manganese [13], although consumption of these foods increased significantly in Middle East populations in the last decades [14]. Hypothetically, the observed differences may occur due to geochemical aspects of manganese deposition. Particularly, manganese deposits and production are located in Africa, Asia, Russia, as well as Latin America, but not Middle East [15].

Although the particular causes of the observed differences are unclear, reduced levels of Co and Mn may significantly contribute to health and education performance. Particularly, it has been demonstrated that children with low Co status are characterized by lower adaptive reserves [16]. In turn, both high and low Mn status (U-shaped) were shown to be associated with impaired intelligence [6]. These findings indirectly corroborate the earlier observed higher rate of impaired adaptive responses in foreign students [17]. Therefore, monitoring and nutritional improvement of trace element status in foreign students may be considered as a useful tool for increasing health and performance in foreign students.

\section{References}

1. M. G. Skalnaya, A. V. Skalny, Essential trace elements in human health: a physician's view (Publishing House of Tomsk State University, Tomsk, 2019).

2. P. J. Magee, M. T. McCann, Proc. Nutr. Soc., 78, 147-149 (2019)

3. P., Chen, J. Bornhorst, M. Aschner, Front. Biosci., 23, 1655-1679 (2019)

4. K. Yamada, Interrelations between Essential Metal Ions and Human Diseases, 295320(Springer, Dordrecht, 2013)

5. L. Leyssens, B. Vinck, C., Van Der Straeten, F. Wuyts, L. Maes, Toxicology, 387, 4356 (2017)

6. B., Milton, D., Krewski, D. R., Mattison, N. A., Karyakina, S., Ramoju, N., Shilnikova et al., Neurotoxicology, 58, 217-225 (2017).

7. A. V., Skalny, M. G., Skalnaya, A. A., Tinkov, E. P., Serebryansky, V. A., Demidov et al. Environ. Monit. Assess., 187, 677 (2015)

8. B. H. Kabki, M. Al-Mohalep, A. A. Kirichuk, Trace Elem. Electroly. 35, 190 (2018)

9. G. N. Schrauzer, K. P. Shrestha, M. F. Flores-Arce. Biol. Trace Elem. Res., 34, 161176 (1992).

10. C. Yajnik, U. Deshmukh, P. Katre, T. Limaye, Vitamin $B_{12}, 131-150$ (CRC Press, 2017).

11. B., Hokin, M. Adams, J. Ashton, H. Louie, Asia Pac. J. Clin. Nutr., 13, 289-291. (2004)

12. S. P., Stabler, \& R. H. Allen, Annu. Rev. Nutr. 24, 299-326 (2004)

13. EFSA. EFSA Journal, 13, 4150 (2015)

14. R. Bahn, S. E. Labban, N. Hwalla. Sustain Sci. 14, 1131-1146 (2019)

15. A. P. Das, L. B. Sukla, N. Pradhan, S. Nayak., Bioresour. Technol., 102, 7381-7387 (2011)

16. V. Detkov, A. Skalny, M. Karganov, A. Cherepov, Y. Medvedeva, M. Glazov, L. Isankina, Technol. Living. Syst., 10, 22-28 (2013)

17. A. A. Kirichuk, I. V. Radysh, A. Y. Chizhov, Human Ecol., 1, 20-25 (2019) 